\title{
Left Ventricular Assist Device Inflow Cannula Position May Contribute to the Development of HeartMate II Left Ventricular Assist Device Pump Thrombosis
}

\author{
Jay K. Bhama, MD, ${ }^{1}$ Aditya Bansal, MD $^{2,3}$ \\ ${ }^{1}$ Department of Surgery, University of lowa Health Care, lowa City, IA ${ }^{2}$ Department of Surgery, Ochsner Clinic Foundation, New Orleans, LA \\ ${ }^{3}$ The University of Queensland School of Medicine, Ochsner Clinical School, New Orleans, LA
}

Background: Pump thrombosis (PT) is a dreaded complication after left ventricular assist device (LVAD) implantation. Problems with inflow cannula (IC) position may precipitate thrombus development. We sought to determine if IC position contributes to the development of PT.

Methods: We conducted a retrospective review of 76 HeartMate II LVAD implants. The angle of the IC (AIC) to the horizontal plane was measured on chest x-rays. Patients who developed PT (PT group) were compared to the remaining patients (control group).

Results: The mean age at implantation was $56 \pm 14$ years, and $82 \%$ of the patients were male. Ten patients (13\%) developed PT. Six (60\%) required device exchange, and 4 (40\%) were managed with anticoagulation and/or thrombolysis. The median AIC for all patients at implantation was $59^{\circ}$ (range, $38^{\circ}-98^{\circ}$; 25 th- 75 th interquartile range, $50^{\circ}-75^{\circ}$ ). In the PT group, the median AIC was larger at the time of PT diagnosis compared to implantation $\left(70^{\circ}\right.$ vs $\left.60^{\circ}, P=0.005\right)$. In the control group, the median AIC was also larger at follow-up compared to implantation $\left(61^{\circ}\right.$ vs $\left.58^{\circ}, P<0.001\right)$ although to a lesser degree than in the PT group. No difference was seen in the median AIC between the PT group and the control group at implantation $\left(60^{\circ}\right.$ vs $58^{\circ}$, respectively; $P=0.668)$ or at follow-up $\left(70^{\circ}\right.$ vs $61^{\circ}$, respectively; $\left.P=0.309\right)$. However, the median AIC at follow-up in the PT group was significantly larger than the median AIC at implantation in the control group $\left(70^{\circ}\right.$ vs $58^{\circ}$, respectively; $\left.P=0.014\right)$.

Conclusion: The HeartMate II LVAD IC position contributes to the development of PT. Regular monitoring of cannula position may help identify patients at risk for this problem.

Keywords: Cannula, chest $x$-ray, thrombosis, ventricular assist device

Address correspondence to Jay K. Bhama, MD, Department of Surgery, University of lowa Health Care, 200 Hawkins Drive, SE500 GH, lowa City, IA 52246. Tel: (319) 356-8875. Email: jay-bhama@uiowa.edu

\section{INTRODUCTION}

Pump thrombosis (PT) is a dreaded and occasionally lethal complication after left ventricular assist device (LVAD) placement. In its mildest forms, PT involves the development of a small thrombus within the rotor of the pump that can be treated with simple augmentation of anticoagulation or thrombolysis. However, in its worst form, PT may involve complete thrombotic occlusion of the blood flow channels that typically mandates reoperation for device exchange. Device exchange is associated with significant risk, including hemorrhage, stroke, air embolism, and death. ${ }^{1,2}$ Because of longer wait times for donor hearts and device approval for destination therapy, the average duration of LVAD support has increased. Consequently, more patients are at risk for pump-related complications such as thrombosis.
Numerous factors may contribute to PT, including the anticoagulation regimen, the presence of hematologic diseases such as hypercoagulable states, and noncompliance with medical therapy. Problems related to the positioning of pump components may also predispose to the development of PT. ${ }^{3}$ Malposition of the inflow cannula (IC) with its orifice facing the intraventricular septum or the ventricular free wall and kinking of the outflow graft are examples of component-related problems. In addition, after implantation, the position of the LVAD components within the body may change over time because of factors such as patient weight gain or loss and ventricular remodeling. When position changes occur, especially with the IC, conditions may be created that precipitate deposition of thrombus along the IC and/or pump rotor and bearings.

The purpose of this study was to determine if the position of the LVAD IC or changes in its position over time 
contribute to the development of $\mathrm{PT}$ in patients receiving LVAD therapy.

\section{METHODS \\ Study Design}

Approval for the study was obtained from the University of Pittsburgh Medical Center Total Quality Council. We conducted a retrospective study of 76 consecutive HeartMate II LVAD (Thoratec Corporation) implants performed from 2004 through 2012. The data were extracted from the University of Pittsburgh Medical Center Cardiothoracic Transplantation Program electronic database of prospectively collected data on all mechanical support patients, supplemented by medical record review. Patients who developed PT as defined below (PT group) were compared to the remaining patients (control group). All patients underwent LVAD implantation using standard techniques with left ventricular apical cannulation for inflow and ascending aortic outflow. Standard practice was to secure the inflow elbow of the device to the thoracic wall using umbilical tape secured to the elbow and a heavy polypropylene suture around the lateral aspect of the costal margin.

\section{Determination of the Angle of the Inflow Cannula}

The angle of the IC (AIC) was measured on routine chest $x$-rays using the Philips iSite Picture Archive and Communication System (Philips Healthcare Informatics) angle measurement tool as demonstrated in Figure 1. The horizontal plane, measured at $90^{\circ}$ from a line visually best fitted to the vertebral bodies of the spine, was used as a reference line. The AIC was measured on the postimplantation chest x-ray and at the time of follow-up. For the PT group, follow-up was upon diagnosis; for the control group, follow-up was at the time of heart transplantation, LVAD explant, death on device, or latest follow-up for patients remaining on device support.

\section{Definition of Pump Thrombosis}

Clinically significant PT was suspected in patients with the following characteristics: significant hemolysis (lactate dehy- drogenase $>600 \mathrm{U} / \mathrm{L}$, plasma free hemoglobin $>40 \mathrm{mg} / \mathrm{dL}$ ), sustained power elevations or abnormal device function, and new-onset heart failure not explained by significant valvular heart disease or right heart dysfunction. PT was confirmed by visualization of thrombus within the pump at the time of device exchange. Patients suspected of having PT based upon laboratory measurements of hemolysis who were not candidates for device exchange were treated with thrombolytic therapy.

\section{Statistical Analysis}

Median values of continuous variables were compared using the median test for unpaired comparisons (ie, intergroup comparisons) and the Wilcoxon signed rank test for paired comparisons (ie, intragroup comparisons). Mean values of continuous variables were compared using paired or unpaired $t$ test. Categorical variables were compared using Fisher exact test. Statistical significance was defined as a two-tailed $P<0.05$. Duration of device support was calculated from the date of implantation to the date of PT, death, transplant, weaning/explant, or the end of the study period.

\section{RESULTS}

A total of 76 patients underwent HeartMate II LVAD placement. The mean age at implantation was $56 \pm 14$ years, and $82 \%$ of patients were male. Ten patients (13\%) developed PT: 6 (60\%) required device exchange, and 4 (40\%) were treated with anticoagulation/thrombolysis. Demographics of the 2 groups are presented in the Table. The patients in both groups were similar with regard to age, sex, body mass index, etiology of heart failure, indication for LVAD implantation, and duration of device support. Only one patient had a preexisting hematologic disorder.

The median AIC for all patients at implantation was $59^{\circ}$ (range, $38^{\circ}-98^{\circ}$; 25th-75th interquartile range, $50^{\circ}-75^{\circ}$ ). Figure 2 depicts intragroup and intergroup comparisons of the median AIC at the time of implantation and follow-up for the control group and the PT group. Paired analysis demonstrated that the median AIC increased significantly in both
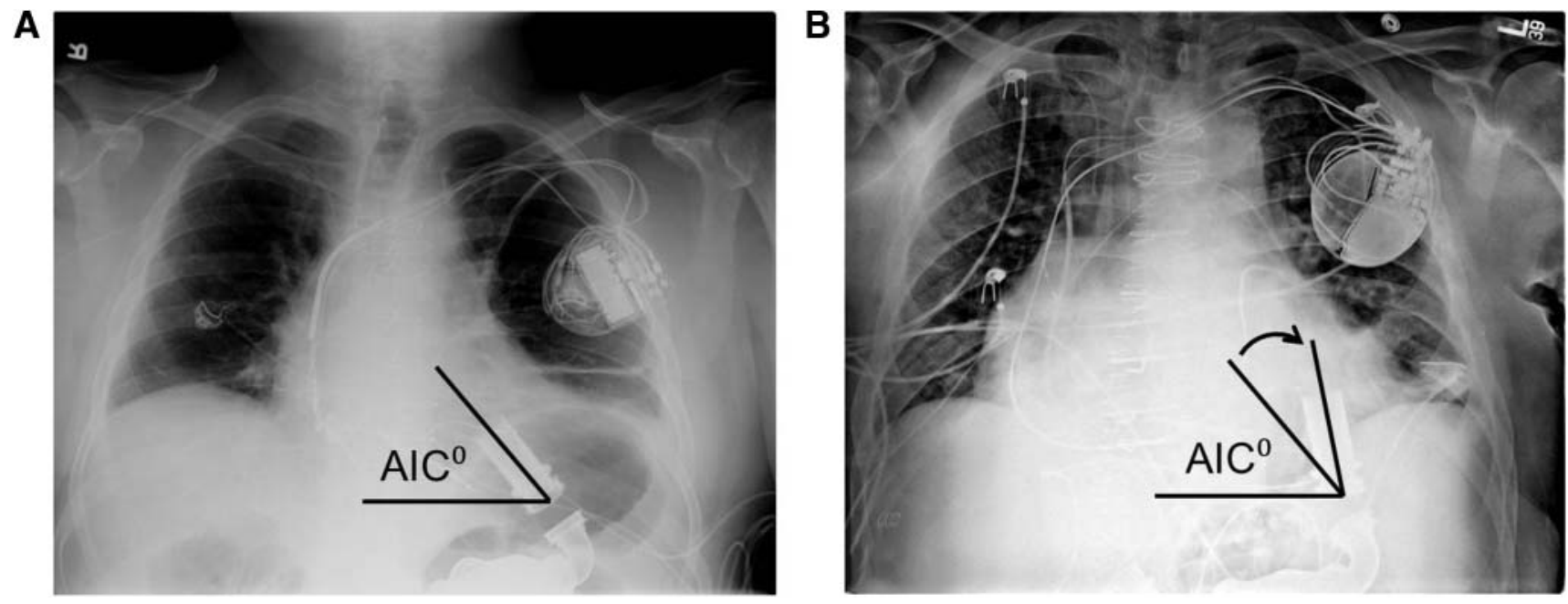

Figure 1. The angle of the inflow cannula (AIC) as measured on routine chest x-ray demonstrates (A) a normal left ventricular assist device (LVAD) cannula position and angle and (B) an abnormal LVAD cannula position and angle in a patient who required device exchange. 
Table. Patient Demographic Data by Group

\begin{tabular}{|c|c|c|c|}
\hline Variable & Control Group $(n=66)$ & Pump Thrombosis Group $(n=10)$ & $P$ Value \\
\hline Mean age, years $\pm S D$ & $57 \pm 15$ & $51 \pm 11$ & 0.259 \\
\hline Male sex & $82 \%$ & $80 \%$ & 1.000 \\
\hline Mean body mass index, $\mathrm{kg} / \mathrm{m}^{2}$ & $29 \pm 7$ & $32 \pm 7$ & 0.318 \\
\hline Etiology of heart failure & & & 1.000 \\
\hline Ischemic & $36(55 \%)$ & $5(50 \%)$ & \\
\hline Nonischemic & $30(45 \%)$ & $5(50 \%)$ & \\
\hline Indication for LVAD implantation & & & 0.299 \\
\hline Bridge to transplant & $28(42 \%)$ & $2(20 \%)$ & \\
\hline Destination therapy/other & $38(58 \%)$ & $8(80 \%)$ & \\
\hline Hematologic disorder & $1(2 \%)$ & $0(0 \%)$ & 1.000 \\
\hline Duration of device support, days & & & 0.734 \\
\hline Median & 201 & 327 & \\
\hline 25th-75th IQR & $59-602$ & $181-509$ & \\
\hline Range & $2-1,470$ & $44-2,036$ & \\
\hline
\end{tabular}

IQR, interquartile range; LVAD, left ventricular assist device.

groups. In the PT group, the median AIC was significantly larger at the time of PT diagnosis compared to implantation $\left(70^{\circ}\right.$ vs $\left.60^{\circ}, P=0.005\right)$. Similarly, for the control group, the median AIC was significantly larger at follow-up compared to implantation $\left(61^{\circ}\right.$ vs $\left.58^{\circ}, P<0.001\right)$. Comparison between the groups found no significant difference in the median AIC at implantation between the PT group and the control group $\left(60^{\circ}\right.$ vs $58^{\circ}$, respectively; $\left.P=0.668\right)$ or at the time of followup between the PT group and the control group $\left(70^{\circ}\right.$ vs $61^{\circ}$, respectively; $P=0.309$ ). The median AIC at follow-up in the PT group was significantly larger than the median AIC at the time of implantation in the control group $\left(70^{\circ} \mathrm{vs}\right.$ $58^{\circ}$, respectively; $\left.P=0.014\right)$. Figure 3 shows box and whisker plots comparing the median AIC for the control group and the PT group at implant and follow-up.

\section{DISCUSSION}

The pertinent finding of this study is that the direction of the LVAD IC position appears to change over time in LVAD-supported patients. We hypothesized that such changes may contribute to the development of PT. Intragroup comparisons using paired analysis demonstrated that the AIC increased significantly in both groups although to a greater degree in the PT group. While we could not demonstrate a significant difference in the AIC and the time of implantation between the 2 groups, the AIC was significantly larger in the PT group at the time of PT diagnosis compared to the control group at the time of implantation. While this intergroup observation does not statistically prove a correlation between the AIC and PT, we propose that the correlation serves as a reasonable suggestion that

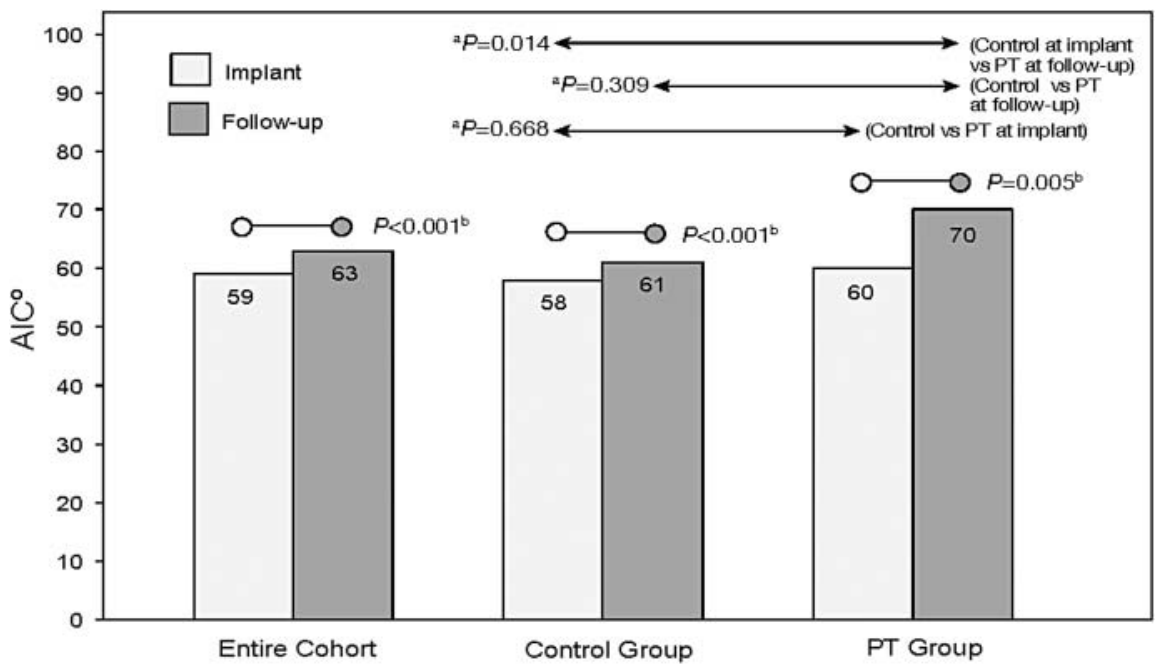

Figure 2. Comparison of the median angle of the inflow cannula (AIC) for the entire cohort, the control group, and the pump thrombosis (PT) group. ${ }^{\text {a }}$ intergroup (unpaired) comparison $P$ values. ${ }^{b}$ Intragroup (paired) comparison $P$ values. 


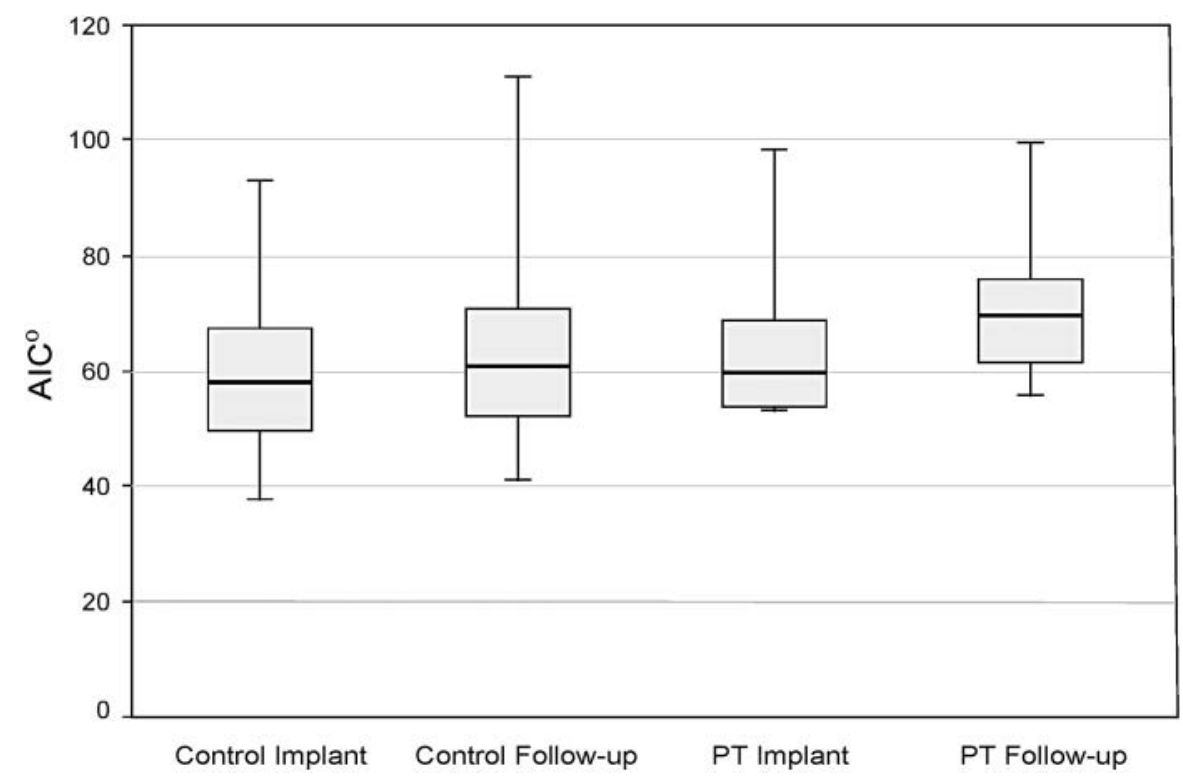

Figure 3. Box and whisker plots comparing the median angle of the inflow cannula (AIC) for the control group and the pump thrombosis (PT) group at implant and follow-up. For each group, the thick black line denotes the median, the upper and lower limits of the box denote the first and third quartile, and the bars denote maximum and minimum values.

changes in the AIC may be related to the development of PT. We suspect that if the initial AIC is suboptimal, subsequent changes, which seem to occur in most patients, may ultimately lead to sufficient deflection of the AIC, resulting in turbulent flow in the IC and predisposing the patient to PT.

Based upon these results, we believe that periodic measurement of the AIC, as a simple mechanism to track changes over time, may be valuable. The authors began looking at the AIC as a rough indicator of whether the orifice of the IC was in the optimal position directed toward the mitral valve. More sophisticated studies are available for delineating the IC position, such as transesophageal echocardiogram (TEE) and contrast-enhanced computed tomography (CT) with 3-dimensional reconstruction. However, these studies are difficult to obtain on a regular basis in ambulatory patients because of cost, invasiveness, and potential nephrotoxicity. Given the results of this study, while it is not common practice, obtaining an intraoperative x-ray prior to chest closure may be useful to help ensure adequate cannula position, using the AIC as a measure. The pump body and IC direction could then be adjusted prior to chest closure. Some centers, including those of the authors, have made securing the elbows of the inflow and outflow cannulae to the thoracic wall standard practice to help align the IC and prevent migration of the pump body. However, despite such measures, the IC position appears to change over time, perhaps as the result of remodeling of the heart itself or changes in body weight and morphology.

The concept that proper positioning of the IC is paramount to good LVAD function is not new. Although a few reports have been published about the impact of IC malposition, ${ }^{4,5}$ the optimal technique for apical IC positioning remains a topic of frequent debate among surgeons. While most surgeons believe that placement just anterior to the apex of the heart is ideal, others recommend placement inferiorly in the diaphragmatic portion of the left ventricle. ${ }^{6}$ This question is difficult to answer because of limited clinical data, but the question has stimulated technologic advancements in cannula design, as well as the development of platforms to study interactions between the cannula and the left ventricle.

In 2010, Fukamachi's group at the Cleveland Clinic described the development of a maneuverable apical cannula that allows for postimplantation adjustment of cannula position. $^{7}$ The design of this cannula permits adjustment of the cannula tip in any plane to a maximum of $15^{\circ}$. In animal studies, this cannula was effective in preventing and controlling suction events related to impingement of the cannula orifice on the ventricular wall. While the design was not intended to be a solution for changes in pump position or ventricular remodeling, in theory, it would be a potential solution in that setting as well.

In 2009, Bhama et al described an in vitro platform for investigating the interactions between the IC and ventricular wall in which intraventricular visualization of the IC was possible in an ovine heart LVAD model. ${ }^{8}$ This model allowed for visualization of how IC position may impact fluid dynamics within the ventricle during LVAD support. Bachman et al demonstrated the feasibility of this model for studying novel IC designs. ${ }^{9}$ In these investigations, impingement of the IC against the ventricular wall significantly reduced LVAD flows, created areas of turbulent flow, and precipitated particle deposition near the IC. The Bachman et al study supports the premise that IC malposition may precipitate thrombus collection within the pump components.

PT often mandates device exchange as seen in $60 \%$ of our patients. Device exchange often involves significant morbidity and mortality. In 2013, Moazami et al reported that PT 
accounted for $36 \%$ of HeartMate II device exchanges in 1,128 patients from the bridge-to-transplant and destination therapy clinical trials for the device. ${ }^{1}$ The overall operative mortality for device exchange was $6.5 \%$, and causes of death included recurrent PT, bleeding, right heart failure, and multiorgan failure. In the Stulak et al single-center review of 422 patients who received LVADs, 45 patients (11\%) required 57 device exchanges. ${ }^{2}$ Operative mortality was $3.5 \%$ for the overall cohort. Actuarial survival in this cohort after exchange was not significantly influenced by the indication for exchange. While these studies demonstrate that exchange can be done safely, the procedure is not benign. Early identification of factors contributing to PT, such as IC position, may lead to timely therapeutic intervention (ie, augmented anticoagulation or transplant listing) and potentially help to avoid the need for exchange with its attendant comorbidities.

Our study has several important limitations. The most important is the variability in routine chest radiography and the fact that the AIC may be influenced by the degree of rotation of the film. The AIC must be measured on a well-performed radiograph with no obvious signs of rotation. Corroboration of the AIC on multiple radiographs is also helpful to clarify suspected changes in IC position. While TEE may provide a more detailed view of the IC position, it is a more complicated procedure, requiring sedation and associated with significant procedural risk compared to a simple chest radiograph. Another major limitation of this study is the small sample size. This limitation makes it difficult to accurately determine if PT was related to changes in position of the IC over time or to malposition at the time of implantation. The fact that the AIC changed significantly between implantation and exchange for all groups suggests that a combination of malpositioning at the time of implantation and change in position over time likely contributes to PT. We believe that large, multiinstitution studies would not only mitigate some of these limitations but would also permit the use of sophisticated analytic methods that might shed further light on this complex association.

\section{CONCLUSION}

We have demonstrated that the position of the LVAD IC changes over time and that this change, in addition to the initial implantation position, may predispose patients to PT. We believe that regular monitoring of the AIC by the simple technique described may help identify patients at risk and potentially indicate the need for further studies (CT or TEE) or preventive therapies such as heightened anticoagulation, transplant, or device repositioning/exchange.

\section{ACKNOWLEDGMENTS}

Aditya Bansal, MD and Jay K. Bhama, MD have received speaking honoraria from Abbott/Thoratec, Pleasanton, CA. Both authors were formerly affiliated with the University of Pittsburgh Medical Center, Dr Bhama from 2006-2014 and Dr Bansal from 2007-2011.

\section{REFERENCES}

1. Moazami N, Milano CA, John R, et al; HeartMate II Investigators. Pump replacement for left ventricular assist device failure can be done safely and is associated with low mortality. Ann Thorac Surg. 2013 Feb;95(2):500-505. doi: 10.1016/j.athoracsur. 2012.09.011.

2. Stulak JM, Cowger J, Haft JW, Romano MA, Aaronson KD, Pagani FD. Device exchange after primary left ventricular assist device implantation: indications and outcomes. Ann Thorac Surg. 2013 Apr;95(4):1262-1267; discussion 1267-1268. doi: 10.1016/j. athoracsur.2012.08.031.

3. Milano CA, Simeone AA, Blue LJ, Rogers JG. Presentation and management of left ventricular assist device inflow cannula malposition. J Heart Lung Transplant. 2011 Jul;30(7):838-840. doi: 10.1016/j.healun.2011.03.003.

4. Sorensen EN, Hiivala NJ, Jeudy J, Rajagopal K, Griffith BP. Computed tomography correlates of inflow cannula malposition in a continuous-flow ventricular-assist device. J Heart Lung Transplant. 2013 Jun;32(6):654-657.

5. Taghavi S, Ward C, Jayarajan SN, Gaughan J, Wilson LM, Mangi AA. Surgical technique influences HeartMate II left ventricular assist device thrombosis. Ann Thorac Surg. 2013 Oct;96(4): 1259-1265. doi: 10.1016/j.athoracsur.2013.05.081.

6. Gregoric ID, Cohn WE, Frazier OH. Diaphragmatic implantation of the HeartWare ventricular assist device. J Heart Lung Transplant. 2011 Apr;30(4):467-470. doi: 10.1016/j.healun. 2010.11.014.

7. Shiose A, Kim HI, Takaseya T, et al. Performance of extracorporeally adjustable ventricular assist device inflow cannula. Ann Thorac Surg. 2010 Nov;90(5):1682-1687. doi: 10.1016/j.athoracsur.2010.06.081.

8. Bhama JK, Bachman TN, Kormos RL, Borovetz H, Antaki JF. Development of an ex vivo ovine ventricular assist device model for intraventricular visualization of the inflow cannula. J Heart Lung Transplant. 2009 Aug;28(8):860-861. doi: 10.1016/j. healun.2009.04.027.

9. Bachman TN, Bhama JK, Verkaik J, Vandenberghe S, Kormos RL, Antaki JF. In-vitro evaluation of ventricular cannulation for rotodynamic cardiac assist devices. Cardiovasc Eng Technol. 2011 Sep;2(3):203-211.

This article meets the Accreditation Council for Graduate Medical Education and the American Board of Medical Specialties Maintenance of Certification competencies for Patient Care, Medical Knowledge, and Practice-Based Learning and Improvement. 